\title{
C3a receptor blockade protects podocytes from injury in diabetic nephropathy
}

\author{
Marina Morigi, ' Luca Perico,' Daniela Corna, ${ }^{1}$ Monica Locatelli, ${ }^{1}$ Paola Cassis, ${ }^{1}$ \\ Claudia Elisa Carminati, ${ }^{1}$ Silvia Bolognini, ${ }^{1}$ Carlamaria Zoja, ${ }^{1}$ Giuseppe Remuzzi, ${ }^{1,2}$ \\ Ariela Benigni, ${ }^{1}$ and Simona Buelli ${ }^{1}$ \\ 'Istituto di Ricerche Farmacologiche Mario Negri IRCCS, Centro Anna Maria Astori, Science and Technology Park Kilometro \\ Rosso, Bergamo, Italy. ““L. Sacco” Department of Biomedical and Clinical Sciences, University of Milan, Milan, Italy.
}

\begin{abstract}
Renal activation of the complement system has been described in patients with diabetic nephropathy (DN), although its pathological relevance is still ill-defined. Here, we studied whether glomerular $\mathrm{C} 3 \mathrm{a}$, generated by uncontrolled complement activation, promotes podocyte damage, leading to proteinuria and renal injury in mice with type 2 diabetes. BTBR ob/ob mice exhibited podocyte loss, albuminuria, and glomerular injury accompanied by C3 deposits and increased C3a and C3a receptor (C3aR) levels. Decreased glomerular nephrin and $\alpha$-actinin4 expression, coupled with integrin-linked kinase induction, were also observed. Treatment of DN mice with a C3aR antagonist enhanced podocyte density and preserved their phenotype, limiting proteinuria and glomerular injury. Mechanistically, ultrastructural and functional mitochondrial alterations, accompanied by downregulation of antioxidant superoxide dismutase 2 (SOD2) and increased protein oxidation, occurred in podocytes and were normalized by C3aR blockade. In cultured podocytes, C3a induced CAMP-dependent mitochondrial fragmentation. Alterations of mitochondrial membrane potential, SOD2 expression, and energetic metabolism were also found in response to C3a. Notably, C3a-induced podocyte motility was inhibited by SS-31, a peptide with mitochondrial protective effects. These data indicate that $\mathrm{C} 3$ a blockade represents a potentially novel therapeutic strategy in DN for preserving podocyte integrity through the maintenance of mitochondrial functions.
\end{abstract}

Conflict of interest: The authors have declared that no conflict of interest exists.

Copyright: () 2020, American Society for Clinical Investigation.

Submitted: July 16, 2019

Accepted: January 29, 2020

Published: March 12, 2020.

Reference information: JCI Insight. 2020;5(5):e131849.

https://doi.org/10.1172/jci.

insight.131849.

\section{Introduction}

Diabetic nephropathy (DN) is one of the main complications of diabetes and is considered the single strongest predictor of mortality $(1,2)$. Renal involvement occurs in about $30 \%$ of diabetic patients, which accounts for more than $40 \%$ of new cases of end-stage renal disease $(3,4)$. The mainstays of current therapy for DN are controlling hyperglycemia and blood pressure and inhibiting the renin angiotensin system, but imperfect renoprotection may occur if pharmacological intervention is begun at an advanced phase of disease $(5,6)$. Thus, novel approaches that target pathogenic pathways other than the renin angiotensin system are needed for diabetic patients who remain at risk of poor renal outcomes. There is compelling evidence from clinical studies that shows that the complement system is activated during progressive nephropathy in diabetes $(7,8)$. Glomerular C3 deposits were observed in kidney biopsies (9), and increased levels of the complement-active fragments $\mathrm{Bb}, \mathrm{C} 4 \mathrm{~d}, \mathrm{C} 3 \mathrm{a}$ and $\mathrm{C} 5 \mathrm{a}$, and soluble $\mathrm{C} 5 \mathrm{~b}-9$ were detected in the urine of patients with DN, compared with those in diabetics without renal involvement (8). Moreover, a tight correlation was demonstrated between urinary $\mathrm{C} 3 \mathrm{a}$ levels and the severity of glomerular lesions, suggesting that C3a may have a pathogenic role in $\mathrm{DN}$ (8). Congruent with these data, the observation that C3a and its receptor $\mathrm{C} 3 \mathrm{aR}$ increased in the glomeruli of diabetic rats and that treatment with a C3aR antagonist partially reduced the endothelial-myofibroblast transition and glomerulosclerotic lesions (10), points to C3a as a crucial trigger of endothelial dysfunction in the progression of $\mathrm{DN}$. Whether the glomerular engagement of the $\mathrm{C} 3 \mathrm{a} / \mathrm{C} 3 \mathrm{aR}$ axis can occur in the neighboring podocytes, impairing their functional integrity and contributing to the development of proteinuria, has not been addressed in DN.

A growing body of evidence suggests that in DN injury to podocytes is a major cause of albuminuria and disease progression toward glomerulosclerosis (11-13). In the diabetic milieu, podocytes undergo phenotypic changes and acquire promigratory and fibrotic features, resulting in their detachment 
from the glomerular basement membrane (GBM), with the consequent development of proteinuria (12, 14-16). Several studies have recognized that there is a close link between podocyte injury and alterations of the mitochondrial functional integrity in DN (17-21). Indeed, changes in mitochondrial dynamics, toward excessive mitochondrial fission and fragmentation, have been shown to impair mitochondrial energy production and to increase oxidative stress in the podocytes of diabetic $d b / d b$ mice (17). In these mice, conditional podocyte-specific deletion of the fission protein dynamin-related protein 1 (Drp1) markedly improved podocyte mitochondrial fitness and slowed the progression of DN (17). The finding that increased mitochondrial oxidative stress was observed in glomerular cells, including podocytes, in diabetic mice further emphasizes the effect that mitochondrial dysfunction has on podocyte injury in DN (18). Since recent work has identified a causal interconnection between the activation of the complement system and derangement of mitochondrial homeostasis in different cellular systems (22-24), a new paradigm is emerging, in which abnormal glomerular complement activation could drive toward podocyte injury through its harmful effect on mitochondrial functions in DN.

Based on all of these considerations, here we sought to investigate in a mouse model of type $2 \mathrm{DN}$ whether (a) glomerular complement activation and modulation of the C3a/C3aR axis occur in podocytes; (b) glomerular C3a was instrumental to podocyte dysfunction associated with the development of albuminuria and glomerular damage, by studying the effect of a C3aR antagonist treatment; and (c) the blockade of $\mathrm{C} 3 \mathrm{aR}$ limited podocyte injury by preserving mitochondrial structural and functional integrity. Furthermore, to prove that $\mathrm{C} 3 \mathrm{a}$ has a detrimental role in mitochondrial dysfunction, cultured human podocytes challenged with $\mathrm{C} 3 \mathrm{a}$ were also examined.

\section{Results}

Complement activation and consequent C3a generation occur in glomeruli of mice with DN. To study the role of the complement system in the development of glomerular damage in DN, we used black and tan, brachyuric (BTBR) ob/ob leptin-deficient mice, a model of type $2 \mathrm{DN}$ characterized by obesity, hyperglycemia, and dyslipidemia (Table 1). Marked staining of C3 was observed in the glomerular tuft of BTBR $o b / o b$ mice at 14 weeks of age compared with WT mice, as assessed by confocal microscopy (Figure 1A). Glomerular C3 activation was accompanied by the generation of $\mathrm{C} 3 \mathrm{a}$ - the complement active fragment known to be produced by the C3 cleavage (25) - in diabetic BTBR ob/ob mice, whereas no C3a staining was found in WT mice (Figure 1B). As shown in Figure 1B, C3a staining was observed in the glomerular vascular tuft and in podocytes of DN mice. Data that showed that increased C3 and C3a staining was also observed in the liver and spleen reflected a systemic complement activation (Supplemental Figure 1; supplemental material available online with this article; https://doi.org/10.1172/ jci.insight.131849DS1). The expression of C3aR, which is known to be present on different renal cells, including podocytes (26-29), was then evaluated. Immunofluorescence analysis in the renal tissue of control mice revealed weak glomerular $\mathrm{C} 3 \mathrm{aR}$ protein expression (Figure 1C) that increased markedly in BTBR ob/ob mice, specifically in podocytes, as revealed by quantification of costaining of C3aR and nestin (Figure 1C). Notably, the increased glomerular expression of C3aR was not a feature specific to BTBR $o b / o b$ mice but was also observed in another model of diabetes induced by streptozotocin (Supplemental Figure 2). In concomitance with the abnormal glomerular complement activation, mice with DN exhibited elevated urinary albumin levels that increased progressively over time (Figure 2A), associated with a significant reduction in both the number of WT1-positive podocytes and their density at 14 weeks of age (Figure 2B). Histological analysis showed increased mesangial matrix expansion and mesangiolysis in DN mice compared with controls (Supplemental Figure 3A) as well as enhanced accumulation of Mac-2-positive monocytes/macrophages in the glomeruli (Supplemental Figure 3B).

C3aR antagonist treatment limits podocyte dysfunction and loss in diabetic mice. The functional role of the glomerular $\mathrm{C} 3 \mathrm{a} / \mathrm{C} 3 \mathrm{aR}$ axis was then investigated by evaluating in $\mathrm{DN}$ mice the effects of $\mathrm{C} 3 \mathrm{aR}$ antagonist treatment starting at 9 weeks of age, when albuminuria was already present. The blockade of C3a with SB290157 markedly limited the progressive rise in albuminuria in diabetic mice to the extent that a $60 \%$ reduction was observed at 14 weeks of age compared with that in vehicle-treated mice (Figure $2 \mathrm{~A}$ ). Notably, inhibition of C3aR significantly limited $(P<0.01)$ podocyte loss and enhanced podocyte density, without affecting glomerular hypertrophy, compared with mice given vehicle (Figure $2 \mathrm{~B}$ ). The protective effect of the $\mathrm{C} 3 \mathrm{aR}$ antagonist on podocyte depletion was also confirmed through the quantification of podocytes labeled with another specific marker, nestin (Supplemental Figure 4). Moreover, treatment with 
Table 1. Systemic and laboratory parameters in BTBR WT and BTBR ob/ob mice at 14 weeks of age

\begin{tabular}{lcccc}
\hline Groups & Body weight $(\mathrm{g})$ & Blood glucose $(\mathrm{mg} / \mathrm{dl})$ & Plasma cholesterol (mg/dl) & Plasma triglycerides (mg/dl) \\
BTBR WT & $34.4 \pm 1.4$ & $135 \pm 7$ & $117 \pm 1$ & $135 \pm 3$ \\
BTBR $o b / o b+$ vehicle & $48.1 \pm 2.9^{\mathrm{A}}$ & $596 \pm 4^{\mathrm{A}}$ & $130 \pm 4^{\mathrm{A}}$ & $238 \pm 26^{\mathrm{A}}$ \\
BTBR $o b / o b+$ C3aR antagonist & $53.9 \pm 0.8^{\mathrm{A}}$ & $588 \pm 10^{\mathrm{A}}$ & $135 \pm 5^{\mathrm{A}}$ & $190 \pm 11^{\mathrm{A}}$
\end{tabular}

Data are expressed as mean \pm SEM (WT, $n=5$; BTBR $o b / o b+$ vehicle, $n=8$; BTBR $o b / o b+$ C3aR antagonist, $n=8) .{ }^{A} P<0.01$ vs. BTBR WT.

SB290157 limited the expansion of the mesangial matrix substantially, decreased mesangiolysis slightly (Supplemental Figure 3A), and reduced the glomerular infiltration of Mac-2-positive monocytes/macrophages observed in DN mice (Supplemental Figure 3B).

To discern whether $\mathrm{C} 3$ a promoted podocyte phenotypic alterations in $\mathrm{DN}$, we assessed the expression of nephrin, a critical component of the slit diaphragm whose expression is instrumental to the maintenance of the glomerular filtration barrier. A significant reduction $(P<0.01)$ in nephrin protein expression was observed in podocytes from BTBR $o b / o b$ mice given vehicle, which exhibited heterogeneous expression with a punctate pattern or loss in focal areas, compared with the typical linear staining observed in podocytes of WT mice (Figure 3A). Treatment with the C3aR antagonist preserved nephrin expression and partially restored its linear distribution (Figure 3A). Since podocyte dysfunction and detachment are often associated with changes in cell adhesion to the GBM and in motility (30), we characterized the expression of $\alpha$-actinin 4 , a specific podocyte protein associated with F-actin and integrins (31). In BTBR $o b / o b$ mice, the expression of $\alpha$-actinin4, which was markedly lower compared with that of WT mice (Figure 3B), was restored by the $\mathrm{C} 3 \mathrm{aR}$ antagonist (Figure $3 \mathrm{~B}$ ). We further evaluated the expression of integrin-linked kinase (ILK), an intracellular signaling molecule, associated with focal adhesion kinase proteins, that regulates podocyte/matrix interaction (32). The results showed faint glomerular staining of ILK in WT mice that increased markedly in BTBR ob/ob mice treated with vehicle (Figure 3C). Notably, blocking C3aR normalized ILK expression to control levels (Figure 3C). Altogether, these data suggest that C3a has a key role in glomerular damage and podocyte dysregulation in DN.

We did not observe any effects from SB290157 on body weight, blood glucose, or cholesterol in DN mice, whereas triglyceride levels were numerically reduced without reaching statistical significance, compared with those in vehicle-treated DN mice (Table 1).

C3a inhibition reduces mitochondrial dysfunction and oxidative stress in podocytes of diabetic mice. Since a number of studies have described a causal link between complement activation and mitochondrial functions in diverse cellular systems (22-24), we investigated whether glomerular C3a was instrumental to mitochondrial damage in injured podocytes in DN mice. Consistent with previous studies (17-21), through ultrastructural analysis we found that BTBR ob/ob mice exhibited structural mitochondrial alterations in podocytes, characterized by swelling of the mitochondrial matrix associated with cristae disarrangement, in contrast with WT mice that had intact, elongated mitochondria (Figure 4A). Notably, C3a blockade with SB290157 treatment almost normalized mitochondrial abnormalities in podocytes of DN mice (Figure 4A). In addition, a marked decrease in the glomerular mitochondrial mass was also found through quantification of the mitochondrial marker voltage-dependent anion channel (VDAC), whose expression was restored by C3aR antagonist (Figure 4B). Alterations in mitochondrial structure and mass were accompanied by changes in glomerular mitochondrial energy metabolism, as revealed by the downregulation of ATP5I (Figure 4C), a subunit of the ATP synthase, reflecting a reduction in oxidative phosphorylation and ATP production. Again, the treatment with C3aR antagonist significantly increased ATP5I expression $(P<0.05$, Figure $4 \mathrm{C})$.

Given that damaged mitochondria contribute to the generation of ROS (33), we then assessed the effects of $\mathrm{C} 3 \mathrm{a}$ inhibition on the expression of the mitochondrial antioxidant enzyme superoxide dismutase 2 (SOD2) and on ROS generation by studying nitrotyrosine staining, a marker of protein oxidation that is dependent on peroxynitrite (34). As shown in Figure 4, D and E, in DN mice the glomerular expression of SOD2 was significantly lower $(P<0.01)$ and was associated with an increased level of nitrotyrosine compared with WT mice, especially at the podocyte level (arrowhead). The inhibition of the C3a/C $3 a R$ axis through the use of SB290157 enhanced podocyte SOD2 expression and reduced nitrotyrosine level to control values (Figure 4, D and E). 
A

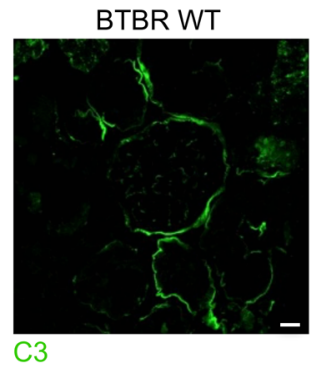

B

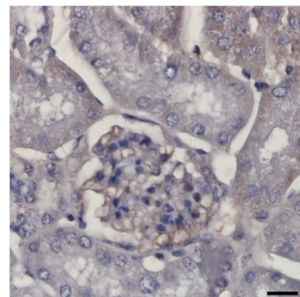

C3a

C
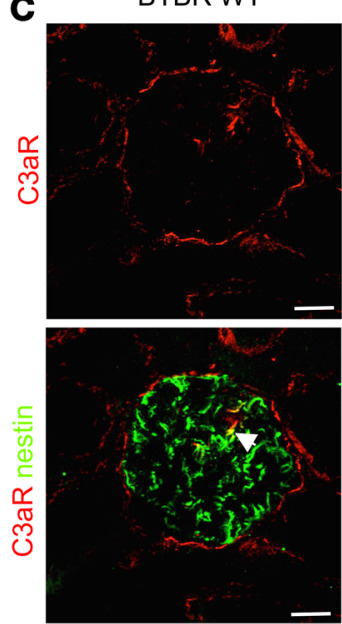

BTBR $o b / o b$

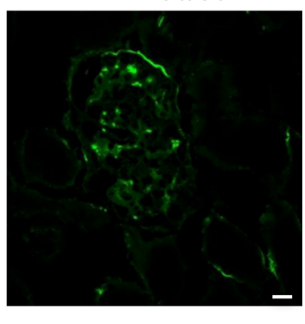

BTBR ob/ob
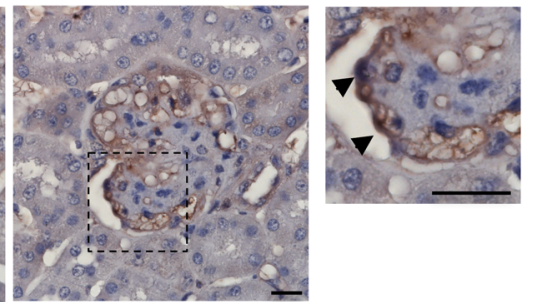

BTBR WT BTBR ob/ob

Figure 1. Glomerular complement activation in mice with type 2 DN. Representative images of glomerular C3 (A) and C3a (B) staining and quantification of C3 (A) in BTBR WT and BTBR ob/ob mice at 14 weeks of age. Results are presented as mean \pm SEM ( $n=4$ mice/group), and unpaired Student's $t$ test was used. ${ }^{*} P<$ 0.05. (B) The image to the right displays the presence of C3a in podocytes (arrowheads). Scale bars: $20 \mu \mathrm{m}$. (C) Representative images and quantification of the double immunofluorescence staining for C3aR (red) and nestin (green) in BTBR WT and BTBR ob/ob mice. The yellow areas (arrowheads) indicate C3aR and nestin colocalization in podocytes. Scale bars: $20 \mu \mathrm{m}$. Results are presented as mean \pm SEM ( $n=4$ mice/group) and unpaired Student's $t$ test was used. ${ }^{* *} P<0.01$.

C3a induces mitochondrial dysfunction in cultured podocytes. To provide evidence that C3a directly affects mitochondrial integrity, cultured human podocytes exposed to C3a were examined. Human podocytes constitutively exhibited a filamentous network of elongated mitochondria in the whole cytoplasm, as revealed by MitoTracker staining of the organelles (Figure 5A). Upon exposure to C3a, massive mitochondrial fragmentation occurred at 6 hours (Figure 5A), along with a remarkable redistribution of these small and round organelles to the perinuclear region of podocytes (Figure 5A). Through the Mitochondrial Network Analysis (MiNA) tool (35), a reduction in mitochondrial networks and area, associated with a decrease in individual mitochondria, was observed in podocytes exposed to C3a (Figure 5B and Supplemental Figure 5A). Then, we studied the expression of Drp1, a cytosolic protein that, once translocated to the outer mitochondrial membrane, tips the balance toward mitochondrial fission and fragmentation (36). Western blot analysis showed that Drp1 accumulated markedly in mitochondria isolated from C3a-treated podocytes compared with control cells (Figure $5 \mathrm{C})$, suggesting that mitochondrial fission is directly induced by C3a.

It has been established that mitochondrial fission and fragmentation are intimately linked with altered redox homeostasis and dissipation of the membrane potential, thus affecting the energy metabolism of the organelles $(17,36,37)$. Consistent with this, we observed that the expression of the mitochondrial antioxidant enzyme SOD2 significantly decreased $(P<0.01)$ at 6 hours in C3a-treated podocytes (Figure 5D), possibly reflecting increased oxidative stress. In this setting, the effect of SB290157 on the recovery of SOD2 expression (Supplemental Figure 5B) confirms that C3a acts through its specific receptor in cultured 
A

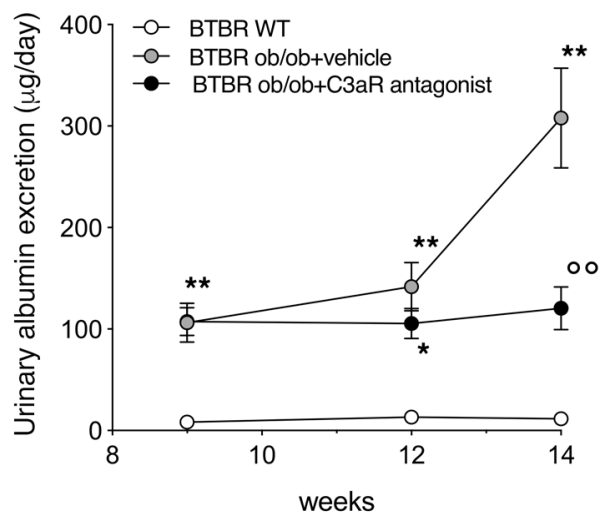

B

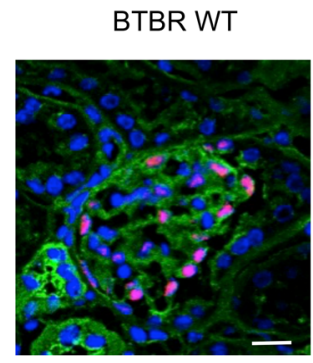

WT1 lectin DAPI
BTBR ob/ob+ vehicle
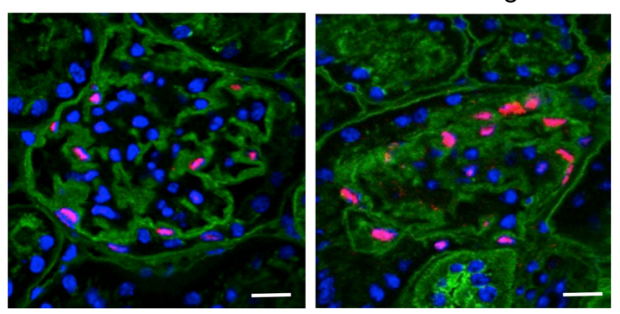

BTBR $a b / o b+$ C3aR antagonist

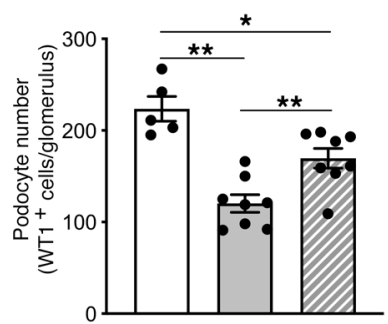

$\square$ BTBR ob/ob+vehicle

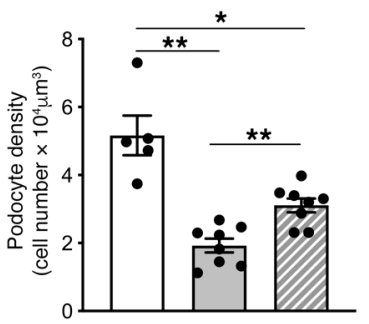

BTBR ob/ob+C3aR antagonist

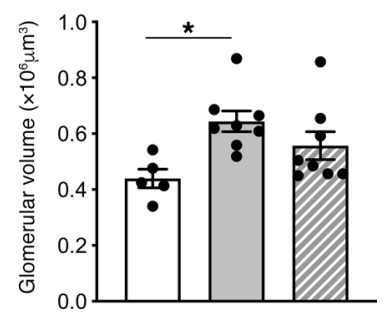

Figure 2. C3aR antagonist reduces albuminuria and limits podocyte loss in BTBR ob/ob mice. (A) Time course of urinary albumin excretion in BTBR WT and BTBR $o b / o b$ mice treated with vehicle or C3aR antagonist $(5 \mathrm{mg} / \mathrm{kg} / \mathrm{d})$. Results are presented as mean \pm SEM (WT mice, $n=5$; BTBR ob) $o b+$ vehicle, $n=8$; BTBR $o b / o b+C 3 a R$ antagonist, $n=8$ ), and ANOVA with Tukey multiple-comparisons test was used. ${ }^{*} P<0.05$, ${ }^{*} P<0.01$ vs. WT, ${ }^{\circ} P<0.01$ vs. vehicle. (B, top) Representative images of WT1 (red) and FITC-WGA-lectin (green) in BTBR WT and BTBR ob/ob mice injected with vehicle or $\mathrm{C3} a \mathrm{R}$ antagonist. Nuclei were counterstained with DAPI (blue). Scale bars: $20 \mu \mathrm{m}$. (B, bottom) Morphometrical estimations of podocyte number (left), podocyte density (middle), and glomerular volume (right). Results are expressed as mean \pm SEM (WT, $n=5$; BTBR ob/ob+vehicle, $n=8$; BTBR ob/ob+C3aR antagonist, $n=8$ ), and ANOVA with Tukey multiple-comparisons test was used. ${ }^{*} P<0.05,{ }^{*} P<0.01$.

podocytes. Mitochondrial polarization was then analyzed by studying JC-1 staining, a dye that shifts its emission spectrum from red to green in a mitochondrial membrane potential-dependent manner. Control podocytes exhibited red-stained intact mitochondria, whereas C3a exposure for 6 hours caused a red-togreen shift in JC-1, reflecting a cytoplasmic distribution of the fluorescent probe (Figure 5E). The quantification of JC-1 staining revealed an $85 \%$ reduction in the red/green area, meaning there was a massive mitochondrial depolarization in C3a-treated podocytes compared with control cells (Figure 5E).

Since energy production in podocytes is regulated by a complex balance between mitochondrial respiration and glycolysis $(38,39)$, we studied the contribution of these two complementary systems in our experimental setting. First, we demonstrated that C3a markedly reduced the total ATP content in podocytes at 15 hours, compared with control cells (Figure 6A). Then, we detected a marked decrease in the mitochondrial activity of citrate synthase, the pace-making enzyme in the first step of the TCA cycle (Figure 6B), as well as a downregulation of ATP5I in C3a-treated podocytes (Figure 6C). On the other hand, in cultured podocytes, $\mathrm{C} 3 \mathrm{a}$ did not modify the expression of phosphofructokinase (PFK; Figure 6D) and the activity of lactate dehydrogenase (LDH; Figure 6E), 2 pivotal enzymes of the glycolytic pathway.

The proof that $\mathrm{C} 3 \mathrm{a}$-dependent mitochondrial damage affects podocyte behavior was provided by testing the effect of SS-31, a mitochondrial protective targeted peptide (40). This agent normalized the fragmentation and the distribution of mitochondria (C3a+SS-31, 26.9\% $\pm 0.2 \%$ versus C $3 a, 43.9 \% \pm 3.9 \%$ of cells with altered mitochondrial pattern, $P<0.01)$ as well as SOD2 downregulation (C $3 a+S S-31,8993 \pm$ 605 fluorescent area/cell versus C3a, $4945 \pm 365$ fluorescent area/cell, $P<0.01$ ) in C3a-treated podocytes. Notably, using a scratch-healing assay, it was observed that podocyte motility increased over time following 

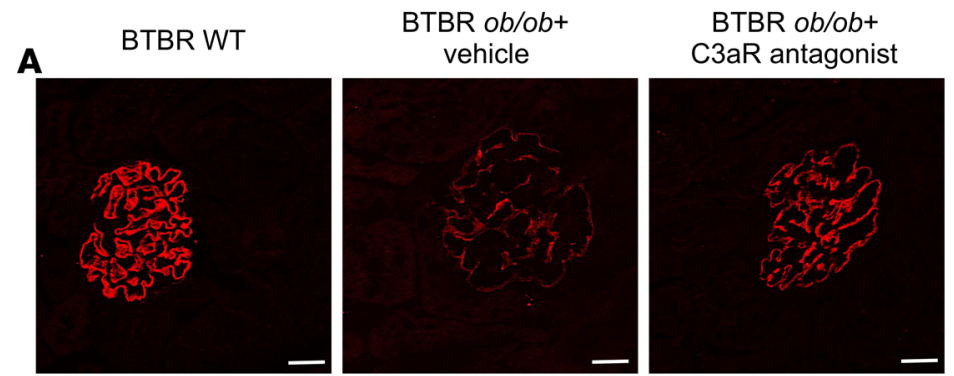

$$
\mathbf{B}^{\mathrm{n}}
$$
nephrin
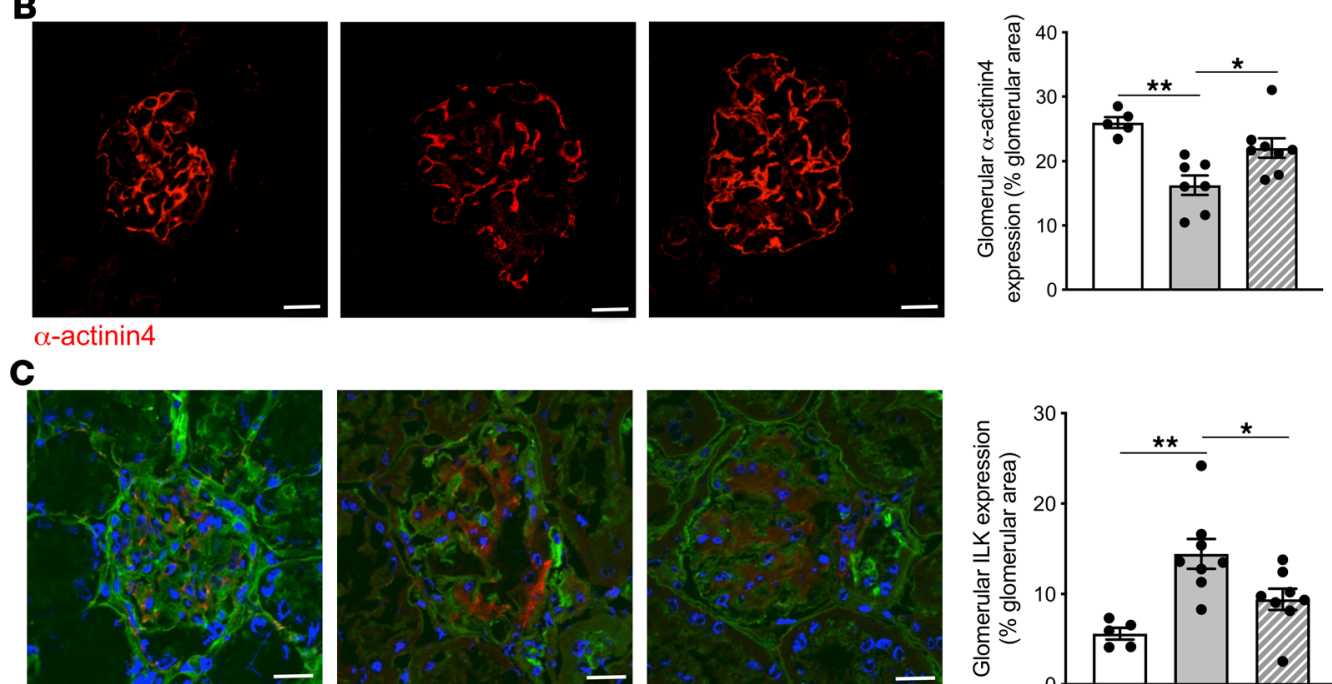

ILK lectin DAPI
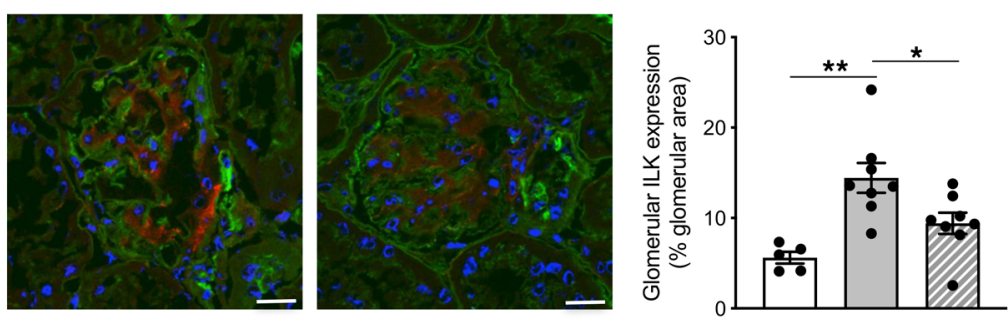

Figure 3. C3aR antagonist limits podocyte dysfunction in BTBR ob/ob mice. Representative images and quantification of nephrin (A), $\alpha$-actinin4 (B), and ILK (C) staining in BTBR WT and BTBR ob/ob mice treated with vehicle or C3aR antagonist. Renal structures and nuclei are stained with FITC-WCA-lectin (green) and DAPI (blue), respectively. Scale bars: $20 \mu \mathrm{m}$. Results are expressed as mean \pm SEM (WT mice, $n=5$; BTBR ob/ob+vehicle, $n=7-8$; BTBR ob/ $o b+C 3 a R$ antagonist, $n=7-8$ ), and ANOVA with Tukey multiple-comparisons test was used. ${ }^{*} P<0.05,{ }^{* *} P<0.01$.

exposure to C3a (Figure 6F) and was inhibited by SS-31. These data suggest that mitochondrial dysfunction, in response to $\mathrm{C} 3 \mathrm{a}$, is instrumental to changes of podocyte phenotype in terms of their motility and adhesive properties to the matrix.

C3a induces mitochondrial fragmentation by affecting cAMP levels in cultured podocytes. It is known that the binding of $\mathrm{C} 3 \mathrm{a}$ to the $\mathrm{G}$ protein-coupled receptor $\mathrm{C} 3 \mathrm{aR}$ induces a remarkable reduction in intracellular cAMP levels in immune cells (41). Moreover, cAMP activators have been described as counteracting mitochondrial fission and apoptosis in adriamycin-injured podocytes (42). Based on these findings, we focused on the role of cAMP signaling in $\mathrm{C} 3 \mathrm{a}$-induced mitochondrial fragmentation, an upstream event leading to changes in mitochondrial polarization, bioenergetics, and oxidative state (36). First, we observed a significant decrease ( $P$ $<0.05)$ in intracellular cAMP content in podocytes exposed to C3a for 3 hours, as compared with unstimulated cells, as assessed by cAMP direct immunoassay (Figure 7A). Then, we evaluated whether mitochondrial structural alterations induced by C3a could be limited by forskolin, an inducer of intracellular cAMP levels. Podocyte mitochondria labeling with MitoTracker revealed that mitochondrial fragmentation in response to $\mathrm{C} 3 \mathrm{a}$ at 6 hours was reduced significantly by the addition of forskolin $(P<0.01$, Figure $7 \mathrm{~B})$, suggesting that $\mathrm{C} 3 \mathrm{a}$ actually induces mitochondrial damage through the modulation of cAMP signaling in podocytes.

\section{Discussion}

In this study, we elected to use the BTBR $o b / o b$ leptin-deficient mice, a well-characterized model of type $2 \mathrm{DN}$ that recapitulates the structural and functional changes of human DN, consisting of sustained hyperglycemia, glomerular hypertrophy, mesangial matrix expansion, podocyte loss, and elevated levels of albuminuria (43). 
A
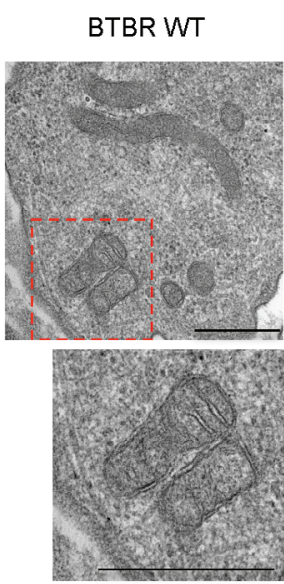

B

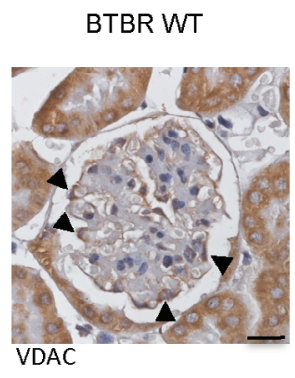

C

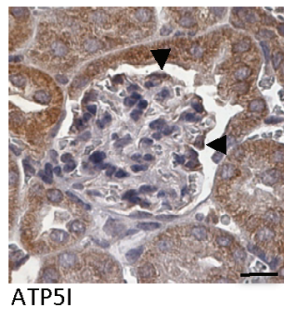

D

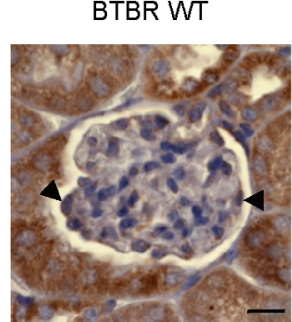
SOD2

E

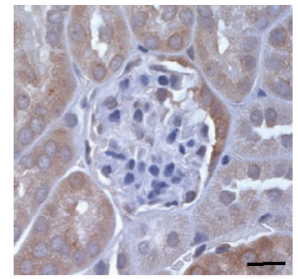

nitrotyrosine
BTBR $o b / o b+$ vehicle
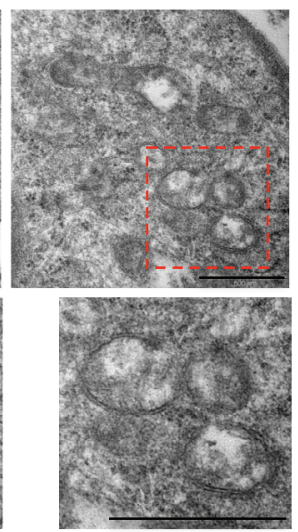

BTBR $o b / o b+$ vehicle
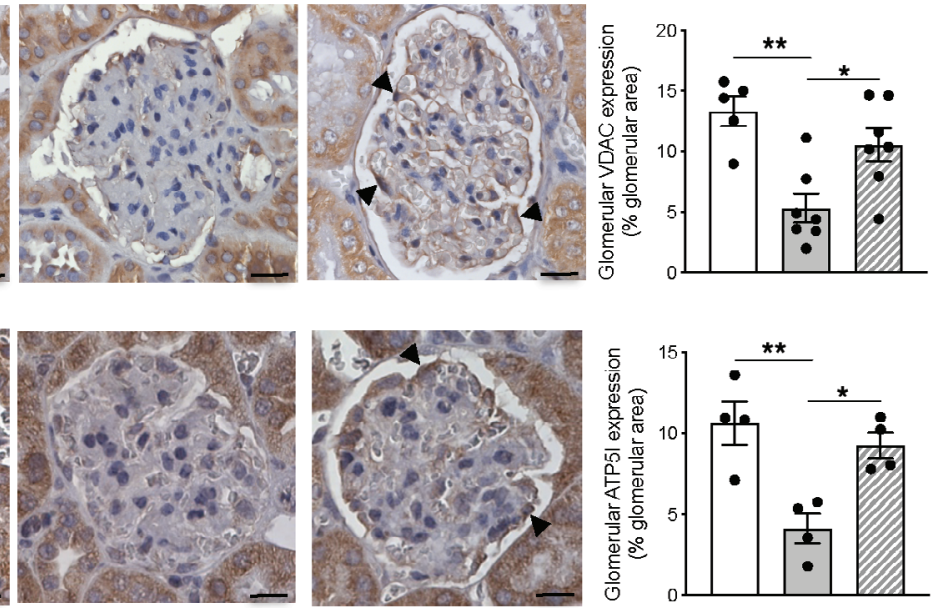

C3aR antagonist
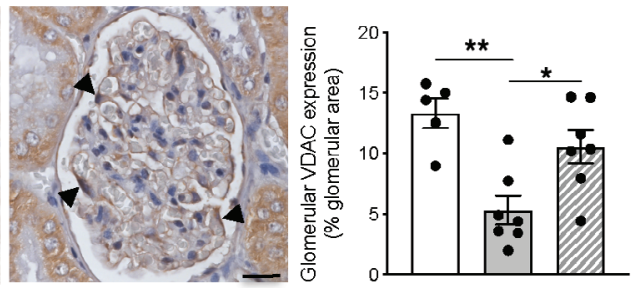

BTBR WT

BTBR ob/ob+vehicle

BTBR $o b / o b+C 3 a R$ antagonist vehicle
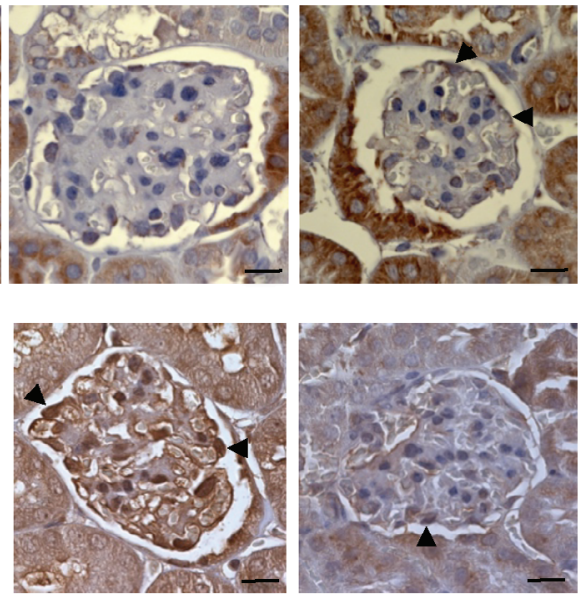

BTBR ob/ob+
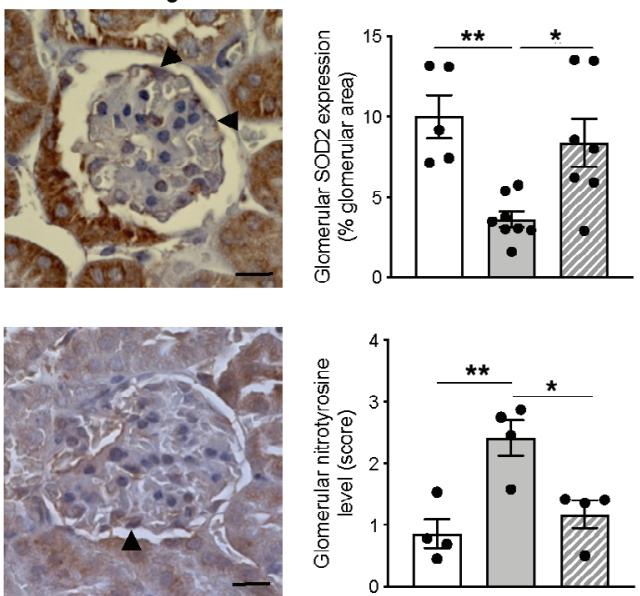
C3aR antagonist
Figure 4. C3aR antagonist protects diabetic mice against mitochondrial dysfunction and oxidative stress in podocytes. (A) Representative transmission electron micrographs showing mitochondrial morphology in podocytes of BTBR WT and BTBR ob/ob mice treated with vehicle or C3aR antagonist ( $n=4$ mice/group). Scale bar: 500 nm. (B-E) Representative images and quantification of glomerular stainings of VDAC (B), ATP5I (C), SOD2 (D), and nitrotyrosine (E) in BTBR WT and BTBR ob/ ob mice treated with vehicle or C3aR antagonist. Podocytes are indicated by arrowheads. Scale bars: 20 $\mu \mathrm{m}$. Results are expressed as mean \pm SEM (VDAC, WT, $n=5$; BTBR ob/ob+vehicle, $n=7$; BTBR $o b / o b+$ C3aR antagonist, $n=7$ ) (ATP5I, $n=4$ mice/group) (SOD2, WT, $n=5$; BTBR $o b / o b+$ vehicle, $n=8$; BTBR $o b / o b+$ C3aR antagonist, $n=7$ ) (nitrotyrosine: $n=4$ mice/group), and ANOVA with Tukey multiple-comparisons test was used. ${ }^{*} P<0.05,{ }^{*} P<0.01$. 
A

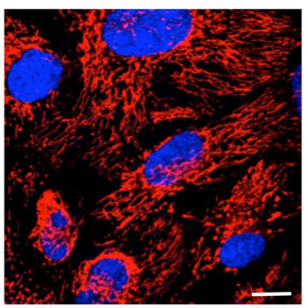

Mitotracker Hoestcht

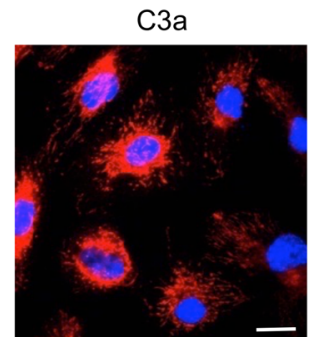

B
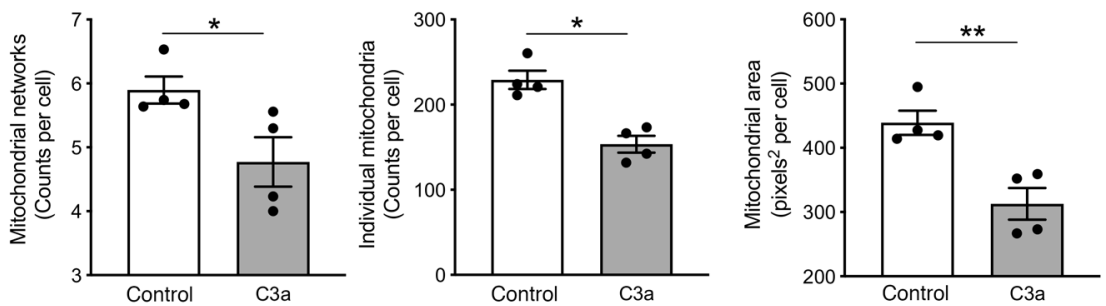

C
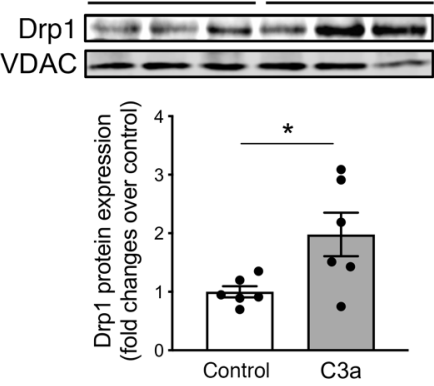

D

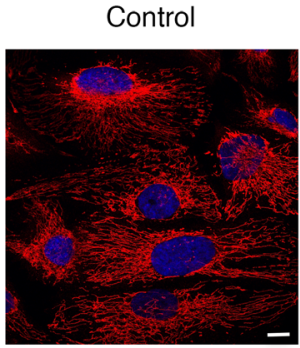

SOD2 DAPI

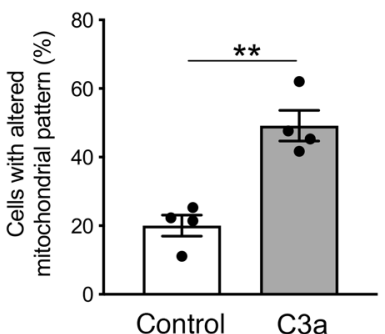

E

Control

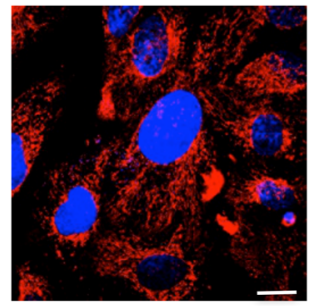

JC-1 mit/JC-1cyt Hoechst

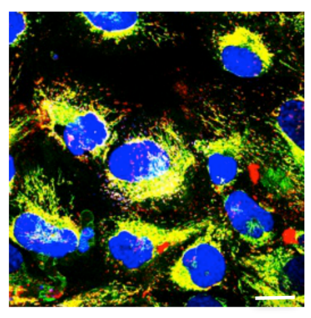

C3a

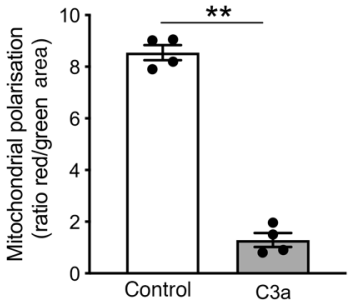

Figure 5. C3a affects mitochondrial functional integrity in cultured podocytes. (A) Representative images of mitochondria labeled with MitoTracker in human podocytes exposed to control medium or $\mathrm{C} 3 \mathrm{a}(1 \mu \mathrm{M})$ for 6 hours. Nuclei were counterstained with Hoechst (blue). Scale bars: $20 \mu \mathrm{m}$. The percentage of podocytes with an altered mitochondrial pattern, in terms of fragmentation and perinuclear redistribution, on total cells per field was quantified. Results are expressed as mean \pm SEM $(n=4)$, and unpaired Student's $t$ test was used. ${ }^{*} P<0.01$. (B) Quantification of mitochondrial networks, individual mitochondria, and mitochondrial area by Mitochondrial Network Analysis (MiNA) tool set in control or C3a-treated podocytes labeled with MitoTracker. Results are expressed as mean \pm SEM $(n=4)$, and unpaired Student's $t$ test was used. ${ }^{*} P<0.05,{ }^{* *} P<0.01$. (C) Western blot and densitometric analysis of Drp1 protein expression in purified mitochondrial fraction isolated from podocytes after incubation with control medium or C3a for 6 hours. VDAC protein expression was used as a sample loading control. Results are expressed as mean \pm SEM $(n=6)$, and unpaired Student's $t$ test was used. ${ }^{*} P<0.05$. (D) Representative images and quantification of SOD2 expression (red) in control or C3a-treated podocytes for 6 hours. Nuclei were stained with DAPI (blue) Scale bars: $20 \mu \mathrm{m}$. Results are expressed as mean \pm SEM $(n=3)$, and unpaired Student's $t$ test was used. ${ }^{*} P<0.01$. (E) Representative images and quantification of mitochondrial membrane potential in podocytes exposed for 6 hours to control medium or C3a. Mitochondrial potential was evaluated by staining with JC-1, a dye sensitive to mitochondrial membrane potential changes that shifts the emission spectrum from red (mitochondrial distribution, JC-1mit) to green (cytoplasmic distribution, JC-1 cyt). Nuclei were counterstained with Hoechst (blue). Mitochondrial membrane potential was evaluated as the ratio between red and green fluorescent areas and normalized for total cells/field. Scale bars: $20 \mu \mathrm{m}$. Results are expressed as mean \pm SEM ( $n=4$ ), and unpaired Student's $t$ test was used. ${ }^{* *} P<0.01$. 
A

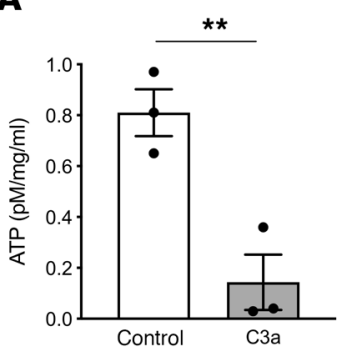

D

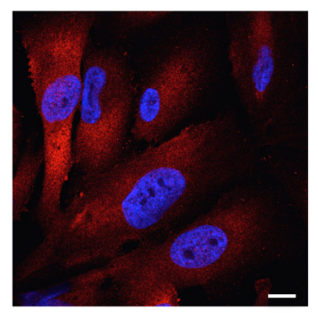

PFK DAPI

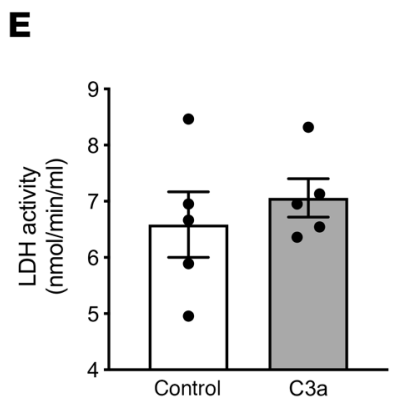

B

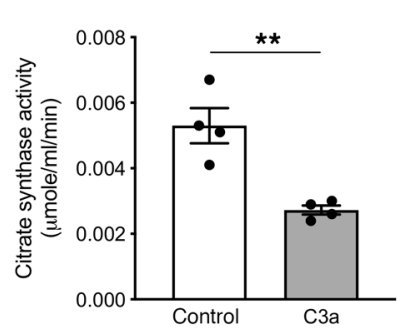

$\mathrm{C} 3 \mathrm{a}$
C

ATP5I

VDAC

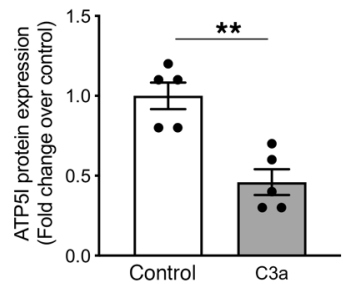

C3a
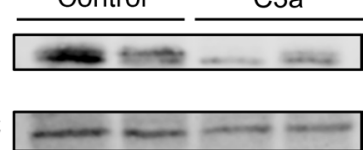

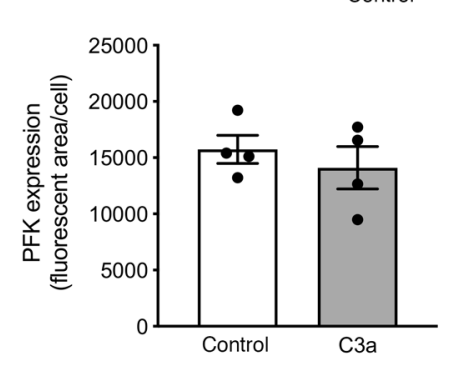

$\mathbf{F}$

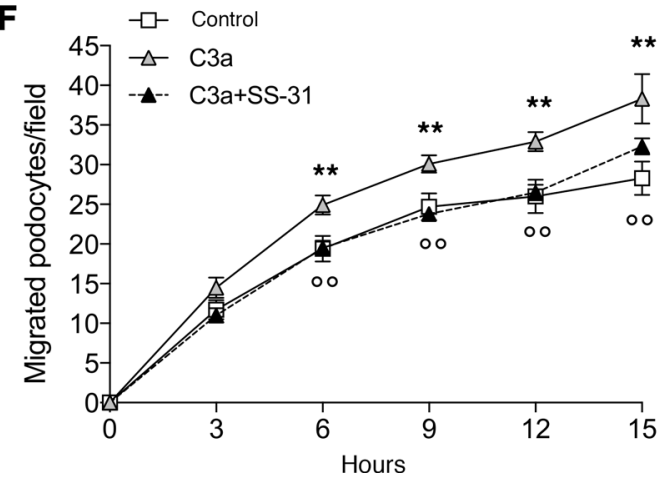

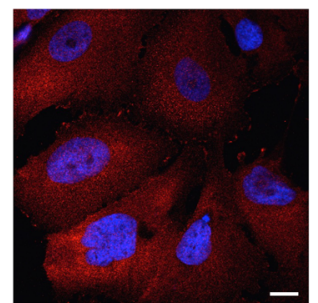

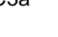

Hours

Figure 6. C3a promotes mitochondrial bioenergetic changes in cultured podocytes, leading to increased podocyte motility. (A and B) Evaluation of ATP content (A) and citrate synthase activity (B) assessed in human podocytes exposed to control medium or $\mathrm{C3a}(1 \mu \mathrm{M})$ for 15 hours. Results are expressed as mean \pm SEM (ATP, $n=3$; citrate synthase, $n=4$ ), and unpaired Student's $t$ test was used. ${ }^{* *} P<$ 0.01. (C) Western blot and densitometric analysis of ATP5I protein expression in purified mitochondrial fraction isolated from podocytes after incubation with control medium or C3a for 15 hours. VDAC protein expression was used as a sample loading control. Results are expressed as mean $\pm \operatorname{SEM}(n=5)$, and unpaired Student's $t$ test was used. ${ }^{*} P<0.01$. (D and E) Representative images and quantification of phosphofructokinase (PFK) expression (D) and lactate dehydrogenase (LDH) activity (E) in control or C3a-treated podocytes for 15 hours. Scale bars: $20 \mu \mathrm{m}$. Results are expressed as mean \pm SEM (PFK, $n=4$; LDH, $n=5$ ), and unpaired Student's $t$ test was used. (F) Podocyte migration evaluated by scratch-healing assay in cells exposed to control medium or $C 3 a(1 \mu \mathrm{M})$ in the presence or absence of SS-31 ( $5 \mu \mathrm{M})$ at different time intervals. Data are expressed as podocytes migrated into the wound track. Results are expressed as mean $\pm \operatorname{SEM}(n=6)$, and ANOVA with Tukey multiple-comparisons test was used. ${ }^{* *} P<0.01$ vs. control, ${ }^{\circ} P$ $<0.01$ vs. C3a.

Here, we demonstrated in BTBR ob/ob mice that (a) increased glomerular staining for C3a and C3aR, upon C3 activation, were associated with podocyte detachment and albuminuria; (b) C3a inhibition by treatment with a C3aR antagonist limited podocyte phenotypic alterations and loss as well as urinary albumin excretion and glomerular injury; and (c) C3a blockade normalized the ultrastructural and functional mitochondrial abnormalities in the podocytes and decreased oxidative stress. In cultured podocytes, C3a had a direct detrimental effect on mitochondrial structural and functional integrity by affecting cAMP levels.

Previous studies have shown that systemic as well as renal complement activation occurred in patients with overt DN $(8,9,44)$. Moreover, glomerular C3aR upregulation already occurred in the early phase of the disease, suggesting that $\mathrm{C} 3 \mathrm{a}$ may contribute to the development of $\mathrm{DN}$ (10). In line with these clinical observations, our findings of systemic complement activation and increased glomerular C3 and C3a staining, together with the upregulation of $\mathrm{C} 3 \mathrm{aR}$ in podocytes of BTBR $o b / o b$ mice, support the hypothesis that the $\mathrm{C} 3 \mathrm{a} / \mathrm{C} 3 \mathrm{aR}$ axis might be part of an unexplored mechanism of podocyte injury in DN. In concomitance with the presence of abnormal glomerular C3a, we found a reduction in podocyte number and density in BTBR $o b / o b$ mice, associated with nephrin and $\alpha$-actinin 4 downregulation, which are consistent with previous findings in human DN showing that downregulation of slit diaphragm and cytoskeletal proteins was closely related to the progression of glomerulopathy and proteinuria $(12,14,45)$. A large body of evidence shows that phenotypic changes in podocytes strongly correlate with their detaching from the GBM, contributing to glomerular dysfunction during DN progression $(11,46,47)$. One of the important hallmarks of the dysfunctional podocytes in the diabetic milieu is the aberrant activation of the integrin/ILK signaling pathways (15, $16,48)$, known to regulate podocyte detachment as well as the Wnt/ $\beta$-catenin signaling pathway (16) and Snail overexpression (12), which directly contribute to podocytopathy (32). Here, we found a marked glo- 
A

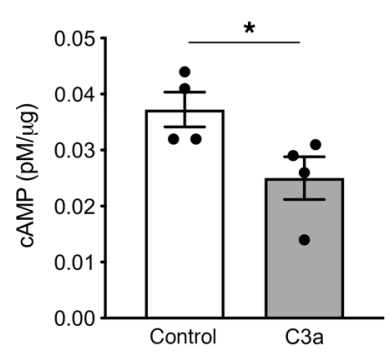

B

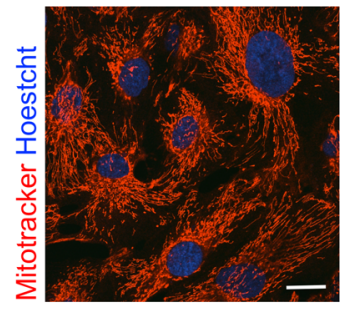

C3a+forskolin

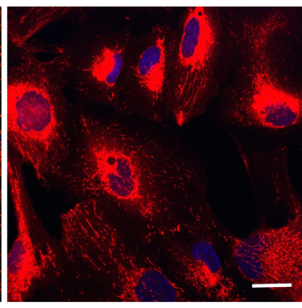

Control+forskolin
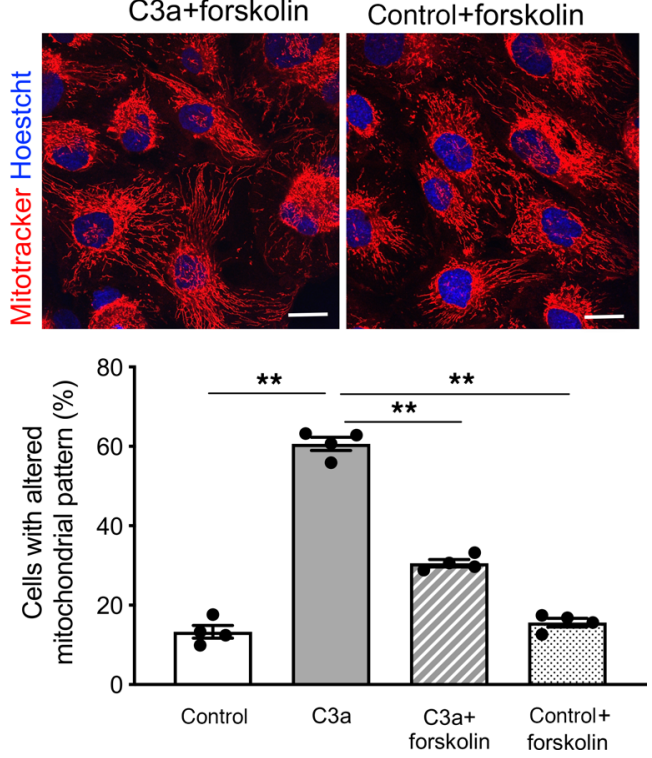

Figure 7. C3a promotes mitochondrial fragmentation by affecting cAMP signaling in cultured podocytes. (A) Detection of intracellular cAMP levels in human podocytes exposed to control medium or C3a (1 $\mu \mathrm{M})$ for 3 hours. Results are expressed as mean $\pm \operatorname{SEM}(n=4)$, and unpaired Student's $t$ test was used. ${ }^{*} P<0.05$. (B) Quantification of mitochondrial alterations in podocytes exposed to control medium, $\mathrm{C} 3 \mathrm{a}(1 \mu \mathrm{M}, 6$ hours), in the presence or absence of the cAMP activator forskolin $(25 \mu \mathrm{M})$, evaluated by MitoTracker staining. Scale bars: $20 \mu \mathrm{M}$. The percentage of podocytes with an altered mitochondrial pattern, in terms of fragmentation and perinuclear redistribution, on total cells per field was quantified. Results are expressed as mean \pm SEM $(n=4)$, and ANOVA with Tukey multiple-comparisons test was used. ${ }^{* *} P<0.01$.

merular ILK overexpression, particularly at podocyte level, in the renal tissue of BTBR ob/ob mice, implying that the interaction between podocyte transmembrane integrins and GBM components was altered in this model. That podocyte dysfunction in DN was functionally linked to glomerular C3a engagement was demonstrated by the treatment of BTBR ob/ob mice with a C3aR antagonist, which had favorable outcomes in terms of reduced podocyte loss, glomerular changes, and urinary albumin excretion. Little is currently known about the renoprotective effects of C3a inhibitors in progressive nephropathy. In this regard, only two reports described the effects of $\mathrm{C} 3 \mathrm{a}$ blockade in rats with type 1 and 2 diabetes induced by streptozotocin alone or in combination with high-fat diet $(10,49)$. These studies showed that inhibition of C3a limited endothelial-mesenchymal transition, an early hallmark of DN (50), and glomerular fibrosis, with very low improvements in renal function and urinary albumin excretion $(10,49)$. To the best of our knowledge, the present study is the first to demonstrate that treatment with a $\mathrm{C} 3 \mathrm{aR}$ antagonist exerted a robust renoprotection and counteracted podocyte phenotypic alterations by preserving nephrin and $\alpha$-actinin 4 expression and limiting glomerular ILK activation in experimental DN. These data follow up on our previous findings in a mouse model of protein overload proteinuria, where we demonstrated that glomerular activation of the complement system via the alternative pathway was instrumental to podocyte depletion and proteinuria (29).

Recent observations indicate that podocyte injury in diabetes is related to mitochondrial damage and oxidative stress $(18,33,51,52)$. To gain insight into the intracellular mechanisms triggered by $\mathrm{C} 3 \mathrm{a}$, different experimental approaches have been used to verify whether C3a blockade counteracted the dysregulation of the mitochondrial homeostasis occurring in damaged podocytes of BTBR $o b / o b$ mice. First, we observed 
that mitochondrial ultrastructural alterations accompanied by the maladaptive oxidative stress response of these organelles were preserved when $\mathrm{C} 3 \mathrm{aR}$ was inhibited. Furthermore, treatment with the $\mathrm{C} 3 \mathrm{aR}$ antagonist normalized the expression of VDAC and ATP synthase, two functional indicators of mitochondrial mass and energy production $(53,54)$, in glomeruli of diabetic mice. All these findings are corroborated by in vitro data showing that $\mathrm{C} 3$ a directly impaired mitochondrial function in human cultured podocytes through the engagement of its specific receptor. A massive fragmentation of the mitochondrial network was found in podocytes challenged with $\mathrm{C} 3 \mathrm{a}$, due to the enhanced recruitment of the fission protein Drp1 to the mitochondrial membranes. It is known that mitochondrial fission and fragmentation are associated with alterations in the redox status and bioenergetics of the organelles $(37,55,56)$. In line with these findings, we showed that C3a induced a remarkable decrease in antioxidant defenses and dissipation of mitochondrial membrane potential, leading to reduced mitochondrial ATP production. Actually, a reduced activity of the two major mitochondrial metabolic routes, the TCA cycle and the oxidative phosphorylation, was found in response to C3a, which was accountable for the impairment of the podocyte energetic metabolism. On the other hand, our data that show that $\mathrm{C} 3$ a reduced total podocyte ATP content without affecting the glycolytic pathway point to the mitochondrial metabolism as the principal target of the detrimental effect of $\mathrm{C} 3 \mathrm{a}$ on the podocyte energetic profile. The hypothesis that disturbances in mitochondrial functions in response to $\mathrm{C} 3 \mathrm{a}$ affect podocyte adhesive properties was demonstrated by in vitro findings that enhanced podocyte motility induced by C3a was normalized by the protective mitochondrial-targeted peptide SS-31. Altogether, these findings support the paradigm that the beneficial effects of $\mathrm{C} 3 \mathrm{a}$ blockade are the result of multiple and complex actions, starting with the maintenance of mitochondrial functional integrity and redox state in podocytes, a prerequisite for counteracting their detachment from the GBM in response to C3a activation in DN mice.

In search of molecular mechanisms through which $\mathrm{C} 3 \mathrm{a}$ causes mitochondrial dysfunction, we focused our attention on the cAMP signaling known to be regulated by the activation of $\mathrm{G}$ protein-coupled receptors, including C3aR $(41,57)$. A number of studies have described that cAMP had a role in the maintenance of podocyte functional integrity $(58,59)$ and that activating the cAMP signaling limited podocyte mitochondrial fission and apoptosis in response to adriamycin (42). Consistent with this, here we demonstrated that the engagement of the $\mathrm{C} 3 \mathrm{a} / \mathrm{C} 3 \mathrm{aR}$ axis on podocytes led to decreased intracellular cAMP levels and that the activator of cAMP, forskolin, counteracted mitochondrial fragmentation, the upstream event leading to alterations in mitochondrial functions. This protective effect of forskolin could be explained by the ability of cAMP to activate AMPK (60), a key regulator of mitochondrial dynamics and biogenesis (61). These data, together with the evidence that Drp1 was recruited in mitochondria in response to C3a, support the idea that there is a detrimental link between C3a and cAMP signaling in triggering mitochondrial damage in podocytes.

In conclusion, this study provides the concept that the glomerular complement activation and the consequent $\mathrm{C} 3 \mathrm{a}$ generation trigger podocyte dysfunction and loss in $\mathrm{DN}$, events that are intrinsically coupled with mitochondrial dysfunction. The translational relevance of these findings rests on the remarkable renoprotective effect of $\mathrm{C} 3 \mathrm{a}$ blockade, a potential new therapeutic approach that could be used for clinical management of DN. Growing evidence highlights the favorable effects of drugs intercepting C3 in preclinical models and in patients with C3 glomerulopathy (62). However, systemic C3 inhibition often raised the concern that a complete shutdown of C3 activity could increase susceptibility to bacterial infections. Thus, $\mathrm{C} 3 \mathrm{a}$ blockade could offer the advantage of avoiding this adverse effect, providing unprecedented opportunities for long-term systemic intervention.

\section{Methods}

\section{In vivo studies}

Animals. Male BTBR Lep ${ }^{o b / o b}$ and BTBR WT mice were obtained from The Jackson Laboratory and were kept in a specific pathogen-free facility with constant temperature on a 12:12-hour light/dark cycle with free access to standard diet and water. At 9 weeks of age, when BTBR $o b / o b$ mice had already developed albuminuria and glomerular damage, animals were randomly allocated to the following groups: group $1(n=8)$, BTBR $o b / o b$ mice given vehicle (saline with DMSO 12.5\%) by intraperitoneal injection twice a day until 14 weeks of age and group $2(n=8)$, BTBR ob/ob mice given the C3aR antagonist SB290157 (Cayman, Chemical) at a dose of 2.5 $\mathrm{mg} / \mathrm{kg}$ by intraperitoneal injection twice a day until 14 weeks of age. Five male BTBR WT mice (group 3) were followed for the same length of time as controls. Mice were euthanized through $\mathrm{CO}_{2}$ inhalation at 14 weeks of age, and their kidneys were collected and processed for analysis. Before sacrifice, mice were housed in meta- 
bolic cages for 24-hour urine collection for albuminuria assessment. Blood samples were collected for glucose, cholesterol, and triglycerides levels measurements. The experimenters were not blind to the treatment, but they were blind for measurement of experimental outcomes. The dose of SB290157 was established on the basis of its effect on the circulating neutrophil count in mice injected with C3a $(60 \mu \mathrm{g} / \mathrm{kg})$, as previously described (63).

Male C57BL/ 6 mice with an initial body weight of 25-30 g received saline or a single intraperitoneal injection of streptozotocin ( $200 \mathrm{mg} / \mathrm{kg}$ body wt; MilliporeSigma) to induce diabetes. Diabetes was confirmed 2 days later based on glucose levels in tail blood. Kidney specimens were observed 4 months after injection of streptozotocin or saline.

Immunohistochemical analyses. For immunofluorescence analysis, OCT-frozen kidney, liver, and spleen sections (3- $\mu \mathrm{m}$ thick) were fixed with cold acetone and incubated with $1 \%$ BSA to block nonspecific sites. For C3, sections were incubated with FITC-conjugated goat anti-mouse C3 (1:200; 55500, Cappel). C3 staining was quantified in 15-20 glomeruli randomly selected from each section using ImageJ 1.40g software $(\mathrm{NIH})$, and data were expressed as a percentage of positive staining on total glomerular area. For C3a, a rabbit anti-C3a antibody (1:100; CAU28002, Biomatik), followed by Cy3-conjugated anti-rabbit secondary antibody (1:100; 711165152, Jackson Immunoresearch Laboratories), was used.

For WT-1, nestin, nephrin, and ILK stainings, OCT-frozen kidney sections were fixed with cold acetone and incubated with 1\% BSA. For nephrin staining, the antigen retrieval with citrate buffer was performed before BSA incubation. The sections were then incubated with the following primary antibodies: rabbit anti-WT-1 (1:400; sc192, Santa Cruz Biotechnology); rat anti-nestin (1:150; ab81462, Abcam); goat anti-nephrin (1:100; sc19000, Santa Cruz Biotechnology); rabbit anti-ILK (1:400; ab76468, Abcam); this was followed by FITC- or Cy3-conjugated secondary antibodies (1:100; 711165152, 705165003, Jackson Immunoresearch Laboratories; F6258, MilliporeSigma). The $\alpha$-actinin4 staining was performed in periodate lysine paraformaldehyde-fixed renal samples (3- $\mu \mathrm{m}$ thick) incubated with $1 \%$ BSA followed by rabbit anti- $\alpha$-actinin 4 antibody (1:300; TA307264 Origene Technologies) and Cy3-conjugated secondary antibody (1:100; 711165152, Jackson ImmunoResearch Laboratories). The quantification of WT-1-positive cells was assessed as described in the Supplemental Methods. Nestin-, $\alpha$-actinin4-, and ILK-positive stainings were quantified in 15-20 glomeruli/section using ImageJ 1.40g software, and data were expressed as a percentage of positive staining on total glomerular area. Nephrin expression was evaluated in 15-20 glomerular cross sections given a score between 0 and 3 ( 0 , no staining; 1 , weak staining along the capillary tuft; 2 , moderate staining along the capillary tuft; 3 , linear and intense staining along the capillary tuft).

For $\mathrm{C} 3 \mathrm{aR}$ expression, double staining was performed with rabbit anti-C3aR antibody (1:100; LS-C382362, Lifespan BioSciences Inc.) and Cy3-conjugated secondary antibody (1:100; 711165152, Jackson Immunoresearch Laboratories), followed by rat anti-nestin (1:150; ab81462, Abcam) and FITC-conjugated secondary antibodies (1:100; F6258, MilliporeSigma). Quantification of the C3aR/nestin toward staining was performed in 15-20 glomeruli/section using ImageJ $1.40 \mathrm{~g}$ software, and data were expressed as a percentage of the $\mathrm{C} 3 \mathrm{aR} /$ nestin costaining area (yellow) on the total glomerular $\mathrm{C} 3 \mathrm{aR}$ area (red).

Nuclei and cell membranes were counterstained with DAPI and rhodamine- or FITC-WGA-lectin, respectively. Negative controls were obtained by omitting the primary antibodies on adjacent sections. Samples were examined under confocal inverted laser microscopy (LSM 510 Meta, Zeiss).

For immunoperoxidase analysis, formalin-fixed, $3-\mu \mathrm{m}$ paraffin-embedded kidney sections were incubated with mouse anti-C3a/C3a des Arg (1:100; ab37230, Abcam) antibody, rabbit anti-nitrotyrosine (1:100; 06-284 Millipore, Merck Life Science S.r.1.) antibody, mouse anti-VDAC (1:250; ab186321, Abcam) antibody, rabbit anti-ATP5I (1:200; HPA035010, MilliporeSigma) antibody, rabbit anti-SOD2 (1:200; HPA001814, MilliporeSigma) antibody, and rat anti-Mac-2 (1:600; MF48000 Molecular Probe, Thermo Fisher Scientific) antibody. The samples were then incubated with rabbit on rodent HRP-Polymer (RMR622G, Biocare Medical), mouse-on-mouse HPR-polymer (MM20G, Biocare Medical) or rat-on-mouse HPR polymer (TR517, Biocare Medical). The staining was visualized by the addition of the betazoid 3,3'diaminobenzidine chromogen kit solutions (BDB2004H, Biocare Medical). Slides were finally counterstained with hematoxylin and observed through light microscopy (ApoTome, Axio Imager Z2, Zeiss). Negative controls were obtained by omitting the primary antibody on adjacent sections. Nitrotyrosine expression was evaluated in 15-20 glomerular cross sections and given a score between 0 and 3 (0, no staining; 1 , faint; 2 , moderate; 3 , intense). The VDAC-, ATP5I-, and SOD2-positive stainings were quantified in 15-20 glomeruli/section with ImageJ 1.40g software, and data were expressed as a percentage of positive staining on total glomerular area. Mac-2-positive monocytes/macrophages were counted in 35-40 glomerular cross sections $(\times 400)$. 


\section{In vitro studies}

Cell culture and incubations. For in vitro experiments, we used the conditionally immortalized human podocyte cell line (provided by P. Mathieson and M.A. Saleem, Children's Renal Unit and Academic Renal Unit, University of Bristol, Southmead Hospital, Bristol, United Kingdom). The methods for podocyte culture, immortalization, and differentiation were based on previously described protocols (64). Briefly, cells were cultured under growth-permissive conditions at $33^{\circ} \mathrm{C}$ in RPMI 1640 medium (21875034, Thermo Fisher Scientific) supplemented with 10\% FBS (10270106, Thermo Fisher Scientific), 1\% ITS 100× (insulin, transferrin, and sodium selenite) (41400045, Thermo Fisher Scientific), and 1\% Pen-Strep (100 U/ml penicillin plus $0.1 \mathrm{mg} /$ $\mathrm{ml}$ streptomycin) (15140122, Thermo Fisher Scientific). To induce differentiation, podocytes were grown on rat collagen type I and maintained in nonpermissive conditions at $37^{\circ} \mathrm{C}$ for at least 12 days. One hour before and during the experiments, podocytes were maintained in control medium (RPMI 1640 medium with 1\% FBS). Podocytes were then incubated with exogenous $1 \mu \mathrm{M}$ C3a (A118, Complement Technology) or control medium for 6 or 15 hours. In selected experiments, podocytes were treated with $1 \mu \mathrm{M} \mathrm{SB} 290157,5 \mu \mathrm{M}$ SS-31 (736992-21, BOC Sciences), or $25 \mu \mathrm{M}$ forskolin (F6886, MilliporeSigma), added 1 hour before and during incubation with C3a. Preliminary experiments showed that these concentrations did not affect podocyte viability, as evaluated through viable cell count using trypan blue dye exclusion (data not shown).

Mitochondrial morphology and membrane potential detection. The fluorescent MitoTracker CmxROS probe (M7512, Molecular Probes, Invitrogen), which covalently binds to mitochondrial proteins by reacting with free thiol groups of cysteine residues regardless of membrane potential, and JC-1 (T3168, Molecular Probes, Invitrogen), a mitochondrial membrane potential sensor, were used to monitor mitochondrial morphology and membrane potential, respectively. Briefly, in the last 30 minutes of the stimuli, MitoTracker $(250 \mathrm{nM})$ or JC-1 $(5 \mu \mathrm{M})$ was added to podocytes plated on type I collagen-coated glass coverslips. At the end of incubations, living cells were rinsed twice in PBS $1 \times$ and examined under confocal inverted laser microscopy (LSM 510 Meta). Nuclei were counterstained with NucBlue Live ReadyProbes (Hoechst, R37605, Thermo Fisher Scientific). The percentage of podocytes with an altered mitochondrial pattern, in terms of fragmentation and perinuclear redistribution, on total cells per field was assessed by staining with MitoTracker in 15 random fields per sample. Mitochondrial morphology was evaluated in 15 random fields per sample using the MiNA tool set (35) in the Fiji software and normalized for the number of Hoechst-positive cells. The quantification of JC-1 red and green areas was performed in 15 random fields per sample by using ImageJ $1.40 \mathrm{~g}$ software, and mitochondrial polarization was expressed as the ratio between red and green fluorescent areas and normalized for the number of Hoechst-positive cells.

\section{Statistics}

The sample size for the in vivo studies was estimated to be at least 4 mice per group to enable the detection of a mean of paired difference in albuminuria of $30 \mu \mathrm{g} / \mathrm{d}$ with an estimated standard deviation of $10(\alpha=$ 0.05 , paired $t$ test, 2 -tailed test) with an $80 \%$ power, based on our previous published data (65). For in vitro experiments, the sample size was estimated to be at least 3 independent experiments to enable the detection of a mean of paired difference in migrated podocytes of 23 cell number/field with an estimated standard deviation of 7 ( $\alpha=0.05$, paired $t$ test, 2-tailed test) with an $80 \%$ power, based on our previous published data (64). Sample/animals were randomly allocated to the experimental groups, and no inclusion/exclusion criteria were used. Quantification analyses were performed in a single-blind fashion.

Data are expressed as mean \pm SEM. Statistical analysis was performed with GraphPad Prism Software. Comparisons were made using the 2-tailed unpaired Student's $t$ test or 1-way ANOVA with Tukey post hoc test, as appropriate. Statistical significance was defined as $P<0.05$. See figure legends for details on the number of biological replicates $(n)$ used.

\section{Study approval}

All procedure involving animals were performed in accordance with institutional guidelines of Istituto di Ricerche Farmacologiche Mario Negri IRCCS, in compliance with national (D.L. no. 26, March 3, 2014) and international laws and policies (directive 2010/63/UE on the protection of animals used for scientific purposes) and were approved by the Institutional Animal Care and Use Committees of the Istituto di Ricerche Farmacologiche Mario Negri IRCCS. 


\section{Author contributions}

MM conceived and designed the study, discussed data, and wrote the manuscript. LP performed experiments, analyzed and discussed data, and wrote the manuscript. DC, S. Bolognini, and ML performed experiments and analyzed data. CEC performed in vitro experiments. PC discussed data. CZ discussed data and revised the manuscript. GR and $\mathrm{AB}$ discussed data and revised the manuscript. S. Buelli conceived and designed the study, performed experiments, analyzed and discussed data, and wrote the manuscript. All authors approved the final version of the manuscript.

\section{Acknowledgments}

The study was supported by European Foundation for the Study of Diabetes/Boehringer Ingelheim European Research Programme in Microvascular Complications of Diabetes 2016 and by Fondazione Cariplo (grant Giovani Ricercatori ref. 2016-0503). We authors wish to thank Patrizio Gagni, Sebastian Villa, and Daniela Rottoli for technical assistance. Manuela Passera helped to prepare the manuscript, and Kerstin Mierke provided English language editing. Antonella Piccinelli helped to prepare the graphical abstract. LP and ML are the recipients of a fellowship from Fondazione Aiuti per la Ricerca sulle Malattie Rare (A.R.M.R.), Bergamo, Italy.

Address correspondence to: Simona Buelli, Istituto di Ricerche Farmacologiche Mario Negri IRCCS, Centro Anna Maria Astori, Science and Technology Park Kilometro Rosso, via Stezzano, 87, 24126 Bergamo, Italy. Phone: 39.035.42131; Email: simona.buelli@marionegri.it.

1. Borch-Johnsen K, Kreiner S. Proteinuria: value as predictor of cardiovascular mortality in insulin dependent diabetes mellitus. Br Med J (Clin Res Ed). 1987;294(6588):1651-1654.

2. Ruggenenti P, Remuzzi G. Time to abandon microalbuminuria? Kidney Int. 2006;70(7):1214-1222.

3. Parving HH, Lewis JB, Ravid M, Remuzzi G, Hunsicker LG, DEMAND investigators. Prevalence and risk factors for microalbuminuria in a referred cohort of type II diabetic patients: a global perspective. Kidney Int. 2006;69(11):2057-2063.

4. Remuzzi G, Schieppati A, Ruggenenti P. Clinical practice. Nephropathy in patients with type 2 diabetes. N Engl J Med. 2002;346(15):1145-1151.

5. Remuzzi G, Macia M, Ruggenenti P. Prevention and treatment of diabetic renal disease in type 2 diabetes: the BENEDICT study. J Am Soc Nephrol. 2006;17(4 Suppl 2):S90-S97.

6. Ruggenenti P, Cravedi P, Remuzzi G. The RAAS in the pathogenesis and treatment of diabetic nephropathy. Nat Rev Nephrol. 2010;6(6):319-330.

7. Flyvbjerg A. The role of the complement system in diabetic nephropathy. Nat Rev Nephrol. 2017;13(5):311-318.

8. Li XQ, Chang DY, Chen M, Zhao MH. Complement activation in patients with diabetic nephropathy. Diabetes Metab. 2019;45(3):248-253.

9. Woroniecka KI, Park AS, Mohtat D, Thomas DB, Pullman JM, Susztak K. Transcriptome analysis of human diabetic kidney disease. Diabetes. 2011;60(9):2354-2369.

10. Li L, et al. C3a and C5a receptor antagonists ameliorate endothelial-myofibroblast transition via the Wnt/ $\beta$-catenin signaling pathway in diabetic kidney disease. Metab Clin Exp. 2015;64(5):597-610.

11. Pagtalunan ME, et al. Podocyte loss and progressive glomerular injury in type II diabetes. J Clin Invest. 1997;99(2):342-348.

12. Gagliardini E, et al. Angiotensin II contributes to diabetic renal dysfunction in rodents and humans via Notch1/Snail pathway. Am J Pathol. 2013;183(1):119-130.

13. Meyer TW, Bennett PH, Nelson RG. Podocyte number predicts long-term urinary albumin excretion in Pima Indians with Type II diabetes and microalbuminuria. Diabetologia. 1999;42(11):1341-1344.

14. Kimura M, et al. Expression of alpha-actinin-4 in human diabetic nephropathy. Intern Med. 2008;47(12):1099-1106.

15. Chen HC, Chen CA, Guh JY, Chang JM, Shin SJ, Lai YH. Altering expression of alpha3beta1 integrin on podocytes of human and rats with diabetes. Life Sci. 2000;67(19):2345-2353.

16. Ying Q, Wu G. Molecular mechanisms involved in podocyte EMT and concomitant diabetic kidney diseases: an update. Ren Fail. 2017;39(1):474-483.

17. Ayanga BA, et al. Dynamin-related protein 1 deficiency improves mitochondrial fitness and protects against progression of diabetic nephropathy. J Am Soc Nephrol. 2016;27(9):2733-2747.

18. Galvan DL, et al. Real-time in vivo mitochondrial redox assessment confirms enhanced mitochondrial reactive oxygen species in diabetic nephropathy. Kidney Int. 2017;92(5):1282-1287.

19. Zhang T, et al. Resveratrol ameliorates podocyte damage in diabetic mice via SIRT1/PGC-1 $\alpha$ mediated attenuation of mitochondrial oxidative stress. J Cell Physiol. 2019;234(4):5033-5043.

20. Szeto HH, Liu S, Soong Y, Alam N, Prusky GT, Seshan SV. Protection of mitochondria prevents high-fat diet-induced glomerulopathy and proximal tubular injury. Kidney Int. 2016;90(5):997-1011.

21. Galvan DL, et al. Drp1S600 phosphorylation regulates mitochondrial fission and progression of nephropathy in diabetic mice. J Clin Invest. 2019;129(7):2807-2823.

22. Ten VS, et al. Complement component c1q mediates mitochondria-driven oxidative stress in neonatal hypoxic-ischemic brain injury. J Neurosci. 2010;30(6):2077-2087. 
23. Martinus RD, Cook CJ. The effect of complement C5a on mitochondrial functions of PC12 cells. Neuroreport. 2011;22(12):581-585.

24. Marshall KM, He S, Zhong Z, Atkinson C, Tomlinson S. Dissecting the complement pathway in hepatic injury and regeneration with a novel protective strategy. J Exp Med. 2014;211(9):1793-1805.

25. Walport MJ. Complement. First of two parts. N Engl J Med. 2001;344(14):1058-1066.

26. Braun MC, et al. Renal expression of the C3a receptor and functional responses of primary human proximal tubular epithelial cells. J Immunol. 2004;173(6):4190-4196.

27. Bao L, Osawe I, Haas M, Quigg RJ. Signaling through up-regulated C3a receptor is key to the development of experimental lupus nephritis. J Immunol. 2005;175(3):1947-1955.

28. Li X, Ding F, Zhang X, Li B, Ding J. The expression profile of complement components in podocytes. Int J Mol Sci. 2016;17(4):471.

29. Morigi M, et al. A previously unrecognized role of C3a in proteinuric progressive nephropathy. Sci Rep. 2016;6:28445

30. Greka A, Mundel P. Cell biology and pathology of podocytes. Annu Rev Physiol. 2012;74:299-323.

31. Dandapani SV, et al. Alpha-actinin-4 is required for normal podocyte adhesion. J Biol Chem. 2007;282(1):467-477.

32. Kang YS, Li Y, Dai C, Kiss LP, Wu C, Liu Y. Inhibition of integrin-linked kinase blocks podocyte epithelial-mesenchymal transition and ameliorates proteinuria. Kidney Int. 2010;78(4):363-373.

33. Sharma K. Mitochondrial dysfunction in the diabetic kidney. Adv Exp Med Biol. 2017;982:553-562.

34. Viappiani S, Schulz R. Detection of specific nitrotyrosine-modified proteins as a marker of oxidative stress in cardiovascular disease. Am J Physiol Heart Circ Physiol. 2006;290(6):H2167-H2168.

35. Valente AJ, Maddalena LA, Robb EL, Moradi F, Stuart JA. A simple ImageJ macro tool for analyzing mitochondrial network morphology in mammalian cell culture. Acta Histochem. 2017;119(3):315-326.

36. Westermann B. Mitochondrial fusion and fission in cell life and death. Nat Rev Mol Cell Biol. 2010;11(12):872-884

37. Willems PH, Rossignol R, Dieteren CE, Murphy MP, Koopman WJ. Redox Homeostasis and Mitochondrial Dynamics. Cell Metab. 2015;22(2):207-218.

38. Ozawa S, et al. Glycolysis, but not mitochondria, responsible for intracellular ATP distribution in cortical area of podocytes. Sci Rep. 2015;5:18575.

39. Abe Y, Sakairi T, Kajiyama H, Shrivastav S, Beeson C, Kopp JB. Bioenergetic characterization of mouse podocytes. Am J Physiol, Cell Physiol. 2010;299(2):C464-C476.

40. Liu S, Soong Y, Seshan SV, Szeto HH. Novel cardiolipin therapeutic protects endothelial mitochondria during renal ischemia and mitigates microvascular rarefaction, inflammation, and fibrosis. Am J Physiol Renal Physiol. 2014;306(9):F970-F980.

41. Li K, et al. Cyclic AMP plays a critical role in C3a-receptor-mediated regulation of dendritic cells in antigen uptake and T-cell stimulation. Blood. 2008;112(13):5084-5094.

42. Li X, et al. cAMP signaling prevents podocyte apoptosis via activation of protein kinase A and mitochondrial fusion. PLoS One 2014;9(3):e92003.

43. Alpers CE, Hudkins KL. Mouse models of diabetic nephropathy. Curr Opin Nephrol Hypertens. 2011;20(3):278-284.

44. Fujita T, et al. Complement-mediated chronic inflammation is associated with diabetic microvascular complication. Diabetes Metab Res Rev. 2013;29(3):220-226

45. Benigni A, et al. Selective impairment of gene expression and assembly of nephrin in human diabetic nephropathy. Kidney Int 2004;65(6):2193-2200.

46. White KE, et al. Podocyte number in normotensive type 1 diabetic patients with albuminuria. Diabetes. 2002;51(10):3083-3089.

47. Coimbra TM, et al. Early events leading to renal injury in obese Zucker (fatty) rats with type II diabetes. Kidney Int. 2000;57(1):167-182.

48. Guo L, Sanders PW, Woods A, Wu C. The distribution and regulation of integrin-linked kinase in normal and diabetic kidneys. Am J Pathol. 2001;159(5):1735-1742.

49. Li L, et al. C3a receptor antagonist ameliorates inflammatory and fibrotic signals in type 2 diabetic nephropathy by suppressing the activation of TGF- $\beta / \operatorname{smad} 3$ and IKB $\alpha$ pathway. PLoS One. 2014;9(11):e113639.

50. Goligorsky MS. Vascular endothelium in diabetes. Am J Physiol Renal Physiol. 2017;312(2):F266-F275.

51. Wang W, et al. Mitochondrial fission triggered by hyperglycemia is mediated by ROCK1 activation in podocytes and endothelial cells. Cell Metab. 2012;15(2):186-200.

52. Galvan DL, Green NH, Danesh FR. The hallmarks of mitochondrial dysfunction in chronic kidney disease. Kidney Int. 2017;92(5):1051-1057.

53. Abu-Hamad S, Sivan S, Shoshan-Barmatz V. The expression level of the voltage-dependent anion channel controls life and death of the cell. Proc Natl Acad Sci USA. 2006;103(15):5787-5792.

54. Neupane P, Bhuju S, Thapa N, Bhattarai HK. ATP synthase: structure, function and inhibition. Biomol Concepts. 2019;10(1):1-10.

55. Mishra P, Chan DC. Metabolic regulation of mitochondrial dynamics. J Cell Biol. 2016;212(4):379-387.

56. Yu T, Robotham JL, Yoon Y. Increased production of reactive oxygen species in hyperglycemic conditions requires dynamic change of mitochondrial morphology. Proc Natl Acad Sci USA. 2006;103(8):2653-2658.

57. Ali DC, et al. $\beta$-Adrenergic receptor, an essential target in cardiovascular diseases. Heart Fail Rev. 2020;25(2):343-354

58. Gao SY, et al. Rho-family small GTPases are involved in forskolin-induced cell-cell contact formation of renal glomerular podocytes in vitro. Cell Tissue Res. 2007;328(2):391-400.

59. He JC, et al. Retinoic acid inhibits HIV-1-induced podocyte proliferation through the cAMP pathway. J Am Soc Nephrol. 2007;18(1):93-102.

60. Yin W, Mu J, Birnbaum MJ. Role of AMP-activated protein kinase in cyclic AMP-dependent lipolysis In 3T3-L1 adipocytes. $J$ Biol Chem. 2003;278(44):43074-43080.

61. Herzig S, Shaw RJ. AMPK: guardian of metabolism and mitochondrial homeostasis. Nat Rev Mol Cell Biol. 2018;19(2):121-135.

62. Ricklin D, Lambris JD. New milestones ahead in complement-targeted therapy. Semin Immunol. 2016;28(3):208-222

63. Hoffmann T, Böttger EC, Baum HP, Messner M, Hadding U, Bitter-Suermann D. In vivo effects of C3a on neutrophils and its contribution to inflammatory lung processes in a guinea-pig model. Clin Exp Immunol. 1988;71(3):486-492.

64. Locatelli M, et al. Shiga toxin promotes podocyte injury in experimental hemolytic uremic syndrome via activation of the alter- 
native pathway of complement. J Am Soc Nephrol. 2014;25(8):1786-1798.

65. Zoja C, et al. Therapy with a selective cannabinoid receptor type 2 agonist limits albuminuria and renal injury in mice with type 2 diabetic nephropathy. Nephron. 2016;132(1):59-69. 